\title{
A NOTE ON EXACT FORMS ON ALMOST COMPLEX MANIFOLDS
}

\author{
Tedi Draghici AND WeIYi Zhang
}

\begin{abstract}
On a compact almost complex manifold $\left(M^{2 n}, J\right)$, the conditions that $J$ admits tamed or compatible symplectic forms are characterized in terms of exact forms. In dimension 4 , it is shown that $J$ admits a compatible symplectic form if and only if $J$ admits tamed symplectic forms with arbitrary $J$-anti-invariant parts.
\end{abstract}

\section{Introduction}

Among other interesting problems raised in [2], Donaldson asked the following question for a compact almost complex 4-manifold $\left(M^{4}, J\right)$ :

Question 1.1. If $J$ is tamed by a symplectic form, is there a symplectic form compatible with $J$ ?

An almost complex structure $J$ on a manifold $M^{2 n}$ is tamed by a symplectic form $\omega$ (and such an $\omega$ is called $J$-tamed), if $\omega$ is $J$-positive, i.e.,

$$
\omega(X, J X)>0, \quad \forall X \in T M, X \neq 0 .
$$

A symplectic form $\omega$ is compatible with $J$ (or $J$-compatible), if $\omega$ is $J$-positive and $J$-invariant, i.e.,

$$
\omega(X, J X)>0 \text { and } \omega(J Y, J Z)=\omega(Y, Z), \quad \forall X, Y, Z \in T M, X \neq 0 .
$$

Question 1.1 was known to have an affirmative answer on $\mathbb{C} P^{2}$ from fundamental works of Gromov [9] and Taubes [17]. This was recently extended by Taubes [16] to all compact 4-manifolds with $b^{+}=1$ and generic $J$. The powerful subvarieties-currentform technique of Taubes has been further refined in [13] for rational 4-manifolds. In particular, it is shown that Question 1.1 is true for all almost complex structures on $\mathbb{C} P^{2} \# \overline{\mathbb{C} P^{2}}$ and $S^{2} \times S^{2}$.

Another approach on the tame-compatible question has been suggested by Donaldson [2]. He showed that, at least in the case $b^{+}=1$, Question 1.1 is a consequence of solving a symplectic analog of the complex Monge-Ampère equation in the CalabiYau theorem (see also [18]). While the symplectic Calabi-Yau problem is still open, significant progress has been achieved in [7,19-21]. Note also that a recent result in [11] shows that the tame-compatible question holds on all compact quotients of four-dimensional Lie groups by discrete subgroups.

Question 1.1 could be asked for higher dimensions as well, but it is known that certain almost complex structures have local obstructions, coming from the structure

Received by the editors December 18, 2011.

1991 Mathematics Subject Classification. xxx.

Key words and phrases. xxx. 
of their Nijenhuis tensor, to admitting compatible symplectic forms (see e.g. [10]). There are no such local obstructions in dimension 4, or for integrable almost complex structures in any dimension. In $[12,15]$ it was asked whether Question 1.1 holds for compact complex manifolds of arbitrary dimensions. As observed in [12], classical results imply that this is true for complex surfaces. Some positive results are known for higher dimensional complex manifolds, e.g. [6,14], but the problem is still open in this case. Note that Theorem 1.4 of [14] implies that any non-Kähler Moishezon manifold is also non-tamed.

After this brief overview of the tame-compatible question, we observe that this note is somewhat complementary to it. The main direction of our four-dimensional result can be paraphrased as saying that if there exists a $J$-compatible symplectic form, then there is great flexibility in constructing $J$-tamed symplectic forms. The precise statement is:

Theorem 1.2. Let $\left(M^{4}, J\right)$ be a compact almost complex manifold. The following are equivalent:

(i) J admits a compatible symplectic form;

(ii) For any $J$-anti-invariant form $\alpha$, there exists a J-tamed symplectic form whose $J$-anti-invariant part is $\alpha$.

The implication $(i i) \Rightarrow(i)$ is obvious, by just taking $\alpha=0$. The implication $(i) \Rightarrow(i i)$ is also elementary, but less obvious. In higher dimensions the statement of Theorem 1.2 is no longer true (see Remark 3.5). A higher dimensional version could be considered Proposition 2.5.

Our results are consequences of some equivalent reformulations of Question 1.1 in terms of certain spaces of exact forms on an almost complex manifold $\left(M^{2 n}, J\right)$.

\section{Reformulations of Donaldson's question}

Let $\left(M^{2 n}, J\right)$ be a compact almost complex manifold. The almost complex structure $J$ acts as an involution, by $\alpha(\cdot, \cdot) \rightarrow \alpha(J \cdot, J \cdot)$, on the space of real $C^{\infty}$-forms $\Omega^{2}$. Thus we have the splitting into $J$-invariant, respectively, $J$-anti-invariant 2 -forms

$$
\Omega^{2}=\Omega_{J}^{+} \oplus \Omega_{J}^{-}
$$

Let $\mathcal{Z}^{k}$ be the space of closed $k$-forms on $M$ and let $\mathcal{Z}_{J}^{ \pm}=\mathcal{Z}^{2} \cap \Omega_{J}^{ \pm}$be the corresponding subspaces of $\mathcal{Z}^{2}$. We will also denote by $\Omega_{J}^{\oplus}$ the cone of $J$-invariant, $J$-positive forms, i.e., the forms $\omega \in \Omega^{2}$ such that $\omega(\cdot, J \cdot)$ defines a Riemannian metric on $M$. It is easy to see that the sets of symplectic forms that are $J$-compatible, respectively, $J$-tamed, denoted respectively by $\mathcal{S}_{J}^{c}, \mathcal{S}_{J}^{t}$, are given by

$$
\mathcal{S}_{J}^{c}=\Omega_{J}^{\oplus} \cap \mathcal{Z}^{2}, \mathcal{S}_{J}^{t}=\left(\Omega_{J}^{\oplus} \oplus \Omega_{J}^{-}\right) \cap \mathcal{Z}^{2} .
$$

With these notations, Question 1.1 becomes: if $\mathcal{S}_{J}^{t} \neq \emptyset$ is $\mathcal{S}_{J}^{c} \neq \emptyset$ as well ?

The conditions that $J$ admits tamed or compatible symplectic forms can be restated in terms of the spaces of exact forms $d \Omega_{J}^{-}, d \Omega_{J}^{+}, d \Omega_{J}^{\oplus}$.

Proposition 2.1. Let $\left(M^{2 n}, J\right)$ be a compact almost complex manifold. Then

(i) $J$ is tamed by a symplectic form if and only if $d \Omega_{J}^{-} \cap d \Omega_{J}^{\oplus} \neq \emptyset$;

(ii) $J$ is compatible with a symplectic form if and only if $d \Omega_{J}^{\oplus}=d \Omega_{J}^{+}$. 
Proof. For $(i)$ suppose that $J$ is tamed by the symplectic form $\omega$, which we decompose into its $J$-invariant and $J$-anti-invariant parts $\omega=\omega_{J}^{+}+\omega_{J}^{-}$. By the tameness assumption, $\omega_{J}^{+} \in \Omega_{J}^{\oplus}$, and since $d \omega=0$, we have $d\left(\omega_{J}^{+}\right)=d\left(-\omega_{J}^{-}\right) \in d \Omega_{J}^{-} \cap d \Omega_{J}^{\oplus}$. Thus, $d \Omega_{J}^{-} \cap d \Omega_{J}^{\oplus} \neq \emptyset$. Showing that $d \Omega_{J}^{-} \cap d \Omega_{J}^{\oplus} \neq \emptyset$ implies that $J$ is tamed is done by just reversing this argument.

For $(i i)$, assuming $d \Omega_{J}^{\oplus}=d \Omega_{J}^{+}$, since $0 \in d \Omega_{J}^{+}$, it follows that there is $\omega \in \Omega_{J}^{\oplus}$ so that $d \omega=0$. For the other implication, note that $\Omega_{J}^{\oplus}-\Omega_{J}^{\oplus}=\Omega_{J}^{+}$, that is any $J$-invariant form can be written as the difference of two $J$-positive forms. Indeed, if $\theta \in \Omega_{J}^{+}$, taking any $\omega \in \Omega_{J}^{\oplus}$, then $(n \omega+\theta)-n \omega=\theta$ and if $n$ is large enough $n \omega+\theta \in \Omega_{J}^{\oplus}$. If, additionally, we can choose $\omega$ with $d \omega=0$, then $d(n \omega+\theta)=d \theta$, so the equality $d \Omega_{J}^{\oplus}=d \Omega_{J}^{+}$is proved.

A direct consequence of Proposition 2.1 is that Question 1.1 can be rephrased as: Question 2.2. Is it true that either $d \Omega_{J}^{-} \cap d \Omega_{J}^{\oplus}=\emptyset$ or $d \Omega_{J}^{\oplus}=d \Omega_{J}^{+}$?

Seemingly unrelated, we could also ask:

Question 2.3. If $\alpha \in \Omega_{J}^{-}$satisfies $d \alpha \in d \Omega_{J}^{\oplus}$, is it true that $d(-\alpha) \in d \Omega_{J}^{\oplus}$ as well?

It turns out that this is also equivalent to Donaldson's question.

Proposition 2.4. Suppose $\left(M^{2 n}, J\right)$ is a compact almost complex manifold. Then Questions 1.1, 2.2 and 2.3 are all equivalent for the given $J$.

Proof. As mentioned already, the equivalence of Questions 1.1 and 2.2 follows from Proposition 2.1. We now show that Question 2.3 implies Question 1.1. Suppose that $J$ is tamed by the symplectic form $\omega=\omega_{J}^{+}+\omega_{J}^{-}$. Then $\omega_{J}^{+} \in \Omega_{J}^{\oplus}$ and $d\left(-\omega_{J}^{-}\right)=$ $d \omega_{J}^{+} \in d \Omega_{J}^{\oplus}$. Question 2.3 implies that there exists a $J$-positive form $\tilde{\omega}_{J}^{+}$such that $d \tilde{\omega}_{J}^{+}=d \omega_{J}^{-}$. This implies $d\left(\omega_{J}^{+}+\tilde{\omega}_{J}^{+}\right)=0$, so $\omega_{J}^{+}+\tilde{\omega}_{J}^{+}$is a symplectic form compatible with $J$.

Conversely, suppose that Question 1.1 is true. If for an $\alpha \in \Omega_{J}^{-}$there exists $\beta \in \Omega_{J}^{\oplus}$ such that $d \alpha=d \beta$, then $\beta-\alpha$ is a symplectic form taming $J$. From Question 1.1, there exists a symplectic form $\omega$ compatible with $J$. Further for a large number $n$, $n \omega-\beta$ is $J$-positive, and $d(n \omega-\beta)=d(-\alpha)$, which implies Question 2.3.

Using essentially the same arguments, we have:

Proposition 2.5. Let $\left(M^{2 n}, J\right)$ be a compact almost complex manifold and assume that $J$ admits tamed symplectic forms. The following are equivalent:

(i) J admits a compatible symplectic form;

(ii) For any $J$-anti-invariant form $\alpha$, if there exists a J-tamed symplectic form with $J$-anti-invariant part $\alpha$, then there also exists a J-tamed symplectic form whose $J$-anti-invariant part is $-\alpha$.

Proof. For the implication $(i i) \Rightarrow(i)$, let $\omega_{1}$ be a $J$-tamed symplectic form and let $\alpha$ be the $J$-anti-invariant part of $\omega_{1}$. From $(i i)$, there exists another $J$-tamed symplectic form $\omega_{2}$ whose $J$-anti-invariant part is $-\alpha$. But then $\omega_{1}+\omega_{2}$ is a $J$ compatible symplectic form, as it has vanishing $J$-anti-invariant part.

Next we show $(i) \Rightarrow(i i)$. Suppose that $\omega$ is a $J$-compatible symplectic form and let $\omega_{1}$ be a $J$-tamed symplectic form with $J$-anti-invariant part $\alpha$. For a large enough 
number $n$, the form $\omega_{2}=n \omega-\omega_{1}$ is $J$-positive and is certainly closed. Thus $\omega_{2}$ is a $J$-tamed symplectic form whose $J$-anti-invariant part is $-\alpha$.

\section{Exact forms on almost complex four-manifolds}

In this section we prove Theorem 1.2 and make a few related remarks. We start by mentioning the sub-groups $H_{J}^{+}, H_{J}^{-}$of $H^{2}(M, \mathbb{R})$,

$$
H_{J}^{ \pm}=\left\{\mathfrak{a} \in H^{2}(M ; \mathbb{R}) \mid \exists \alpha \in \mathcal{Z}_{J}^{ \pm} \text {such that }[\alpha]=\mathfrak{a}\right\}
$$

and their dimensions $h_{J}^{ \pm}=\operatorname{dim}\left(H_{J}^{ \pm}\right)$, which will appear occasionally in this section. The reader can consult the references for more on these subgroups $[1,3-5,8,12]$. Here we just recall the particularity of dimension 4: for any compact almost complex 4-manifold $\left(M^{4}, J\right)$ the subgroups $H_{J}^{+}, H_{J}^{-}$induce a direct sum decomposition of $H^{2}\left(M^{4}, \mathbb{R}\right)$. In particular, $h_{J}^{+}+h_{J}^{-}=b_{2}$, where $b_{2}$ is the second Betti number of $M^{4}$. These facts are no longer true in higher dimensions for general almost complex structures. If $b^{+}$(resp. $b^{-}$) are the "self-dual" (resp. "anti-self-dual") Betti numbers of a compact manifold $\left(M^{4}, J\right)$, it is also known that $h_{J}^{-} \leq b^{+}, h_{J}^{+} \geq b^{-}$, with the inequalities being strict if $J$ is tamed by a symplectic form [3].

The key tool in obtaining Theorem 1.2 is the following observation.

Proposition 3.1. Let $\left(M^{4}, J\right)$ be a compact almost complex four-manifold. Then

$$
d \Omega_{J}^{-} \subsetneq d \Omega^{2}=d \Omega_{J}^{+}
$$

Moreover, the quotient space $\left(d \Omega^{2}\right) /\left(d \Omega_{J}^{-}\right)$is always infinite-dimensional.

Proof. Let $g$ be a Riemannian metric compatible with $J$ and let $\omega$ denote the fundamental form of $(g, J)$. It is well known that

$$
\Omega_{J}^{+}=C^{\infty}(M) \omega \oplus \Omega_{g}^{-}, \Omega_{g}^{+}=C^{\infty}(M) \omega \oplus \Omega_{J}^{-},
$$

where $\Omega_{g}^{ \pm}$denote the spaces of $g$-self-dual (resp. anti-self-dual) two-forms. The relation $d \Omega^{2}=d \Omega_{J}^{+}$is then an immediate consequence of:

Lemma 3.2. Suppose $\left(M^{4}, g\right)$ is a compact Riemannian four-manifold. Then

$$
d \Omega_{g}^{+}=d \Omega_{g}^{-}=d \Omega^{2} .
$$

Proof of Lemma 3.2. By Hodge decomposition, any two-form $\alpha$ is written as $\alpha=$ $\alpha_{h}+d \alpha_{1}+* d \alpha_{2}$, where the terms are respectively the harmonic, the exact and the co-exact parts of $\alpha$. Then it is clear that

$$
d \alpha=d * d \alpha_{2}=d\left(d \alpha_{2}+* d \alpha_{2}\right)=d\left(-d \alpha_{2}+* d \alpha_{2}\right) .
$$

But $\left(d \alpha_{2}+* d \alpha_{2}\right)$ is a self-dual form and $\left(-d \alpha_{2}+* d \alpha_{2}\right)$ is anti-self-dual, thus the lemma is proved.

To finish the proof of the proposition it remains to verify the claim about the dimension of the quotient $\left(d \Omega^{2}\right) /\left(d \Omega_{J}^{-}\right)$. Let $\mathcal{H}_{g}^{+}$be the space of harmonic self-dual forms and let

$$
\mathcal{T}_{g}=\left\{f \in C^{\infty}(M) \mid \exists \alpha \in \mathcal{H}_{g}^{+},<\omega, \alpha>=f\right\}
$$


Since $\mathcal{H}_{g}^{+}$is finite-dimensional, $\mathcal{T}_{g}$ is a finite-dimensional subspace of $C^{\infty}(M)$. With the notations above, $\operatorname{dim}\left(\mathcal{T}_{g}\right)=b^{+}-h_{J}^{-}$. It is clear that

$$
\mathcal{H}_{g}^{+}+\Omega_{J}^{-}=\mathcal{T}_{g} \omega \oplus \Omega_{J}^{-}
$$

This immediately implies

$$
d \Omega_{J}^{-}=d\left(\mathcal{H}_{g}^{+}+\Omega_{J}^{-}\right)=d\left(\mathcal{T}_{g} \omega \oplus \Omega_{J}^{-}\right) .
$$

Moreover, it can be easily seen more: for any $\beta \in \Omega_{g}^{+}, d \beta \in d \Omega_{J}^{-}$if and only if $\beta \in \mathcal{H}_{g}^{+}+\Omega_{J}^{-}=\mathcal{T}_{g} \omega \oplus \Omega_{J}^{-}$. This implies that the map

$$
C^{\infty}(M) \rightarrow d \Omega_{g}^{+}, \quad f \mapsto d(f \omega)
$$

induces an isomorphism between the quotient spaces $C^{\infty}(M) / \mathcal{T}_{g}$ and $\left(d \Omega_{g}^{+}\right) /\left(d \Omega_{J}^{-}\right)$. It follows that the inclusion $d \Omega_{J}^{-} \subset d \Omega_{g}^{+}$is strict and that the quotient is infinitedimensional.

Remark 3.3. Note that Proposition 3.1 can be rephrased in terms of currents. A consequence is that each homology class in $H_{+}^{J}$ has infinitely many $J$-invariant closed representatives, while each class in $H_{-}^{J}$ has a unique $J$-anti-invariant representative. Here $H_{ \pm}^{J} \subset H_{2}(M ; \mathbb{R})$ are the $J$-(anti)-invariant homology groups defined by currents (see $[3,12])$. This should be compared with the fact that each cohomology class in $H_{J}^{-}$, in dimension 4 , has a unique (necessarily harmonic) $J$-anti-invariant representative.

Combining Propositions 2.1 and 3.1, we get:

Proposition 3.4. For a compact almost complex four-manifold $\left(M^{4}, J\right), J$ admits a compatible symplectic structure if and only if $d \Omega_{J}^{\oplus}=d \Omega^{2}$.

The proof of Theorem 1.2 is now immediate.

Proof of Theorem 1.2. The implication $(i i) \Rightarrow(i)$ follows by taking $\alpha=0$, as mentioned in the introduction. For the other direction, let $\alpha \in \Omega_{J}^{-}$and use Proposition 3.4 to get $\beta \in \Omega_{J}^{\oplus}$ so that $d \beta=d(-\alpha)$. Then $\omega=\beta+\alpha$ is a $J$-tamed symplectic form whose $J$-anti-invariant part is $\alpha$.

Remark 3.5. (i) With the notations above, Theorem 1.2 can be restated as: $\mathcal{S}_{J}^{c} \neq \emptyset$ if and only if the projection map $\Omega_{J}^{\oplus} \oplus \Omega_{J}^{-} \rightarrow \Omega_{J}^{-}$when restricted to $\mathcal{S}_{J}^{t}$ is still onto.

(ii) In [12], Li and Zhang found the precise relationship between the tame and compatible cohomology cones. Denoted by $\mathcal{K}_{J}^{t}, \mathcal{K}_{J}^{c}$, these are, respectively, the images of $\mathcal{S}_{J}^{t}$ and $\mathcal{S}_{J}^{c}$ under the natural map $\mathcal{Z}^{2} \rightarrow H^{2}(M, \mathbb{R})$. In dimension 4 , the main result of $[12]$ is:

$$
\text { if } \mathcal{S}_{J}^{c} \neq \emptyset \text {, then } \mathcal{K}_{J}^{t}=\mathcal{K}_{J}^{c}+H_{J}^{-} .
$$

This is valid in higher dimensions as well under the additional assumption $H_{J}^{+}+H_{J}^{-}=$ $H^{2}(M, \mathbb{R})$ (which holds automatically in dimension 4 , see [3]).

(iii) Theorem 1.2 and Proposition 3.1 are no longer true in dimension higher than 4. Indeed, if $J$ is any complex structure on a compact manifold of dimension 6 or higher, $d \Omega_{J}^{-}$cannot be a subset of $d \Omega_{J}^{+}$. This is because a form $\beta \in \Omega_{J}^{-}$is written as $\beta=\alpha+\bar{\alpha}$, with $\alpha$ a complex form of type $(2,0)$, hence, generically, $d \beta$ contains terms of type $(3,0)$ and $(0,3)$. But $\Omega_{J}^{+}=\left[\Omega_{J}^{1,1}\right]_{\mathbb{R}}$, so for an integrable $J, d \Omega_{J}^{+} \subset\left[\Omega_{J}^{2,1} \oplus \Omega_{J}^{1,2}\right]_{\mathbb{R}}$. 
As a further application of Proposition 3.1, we compute the cohomology of some $J$ modified de Rham-type of complexes. Let us denote by $d_{J}^{+}$(resp. $d_{J}^{-}$) the composition of the differential $\Omega^{1} \stackrel{d}{\longrightarrow} \Omega^{2}$ with the projection $\Omega^{2} \rightarrow \Omega_{J}^{+}\left(\right.$resp. $\Omega^{2} \rightarrow \Omega_{J}^{-}$). Note that both $\operatorname{Ker}\left(d_{J}^{-}\right)$and $\operatorname{Ker}\left(d_{J}^{+}\right)$contain the space of closed 1-forms $\mathcal{Z}^{1}$. Replacing the $\Omega^{1}$ and $\Omega^{2}$ terms in the de Rham differential complex, we consider the following $J$-modified complexes:

$$
\begin{aligned}
& 0 \longrightarrow \Omega^{0} \stackrel{d}{\longrightarrow} \operatorname{Ker}\left(d_{J}^{-}\right) \stackrel{d}{\longrightarrow} \Omega_{J}^{+} \stackrel{d}{\longrightarrow} \Omega^{3} \stackrel{d}{\longrightarrow} \Omega^{4} \longrightarrow 0, \\
& 0 \longrightarrow \Omega^{0} \stackrel{d}{\longrightarrow} \operatorname{Ker}\left(d_{J}^{+}\right) \stackrel{d}{\longrightarrow} \Omega_{J}^{-} \stackrel{d}{\longrightarrow} \Omega^{3} \stackrel{d}{\longrightarrow} \Omega^{4} \longrightarrow 0 .
\end{aligned}
$$

The following are immediate observations (using also Proposition 3.1).

Proposition 3.6. Suppose $\left(M^{4}, J\right)$ is a compact almost complex four-manifold.

(i) The group $H_{J}^{+}$(resp. $H_{J}^{-}$) is the cohomology group at $\Omega_{J}^{+}$-level for the complex (3.4) (resp. at $\Omega_{J}^{-}$-level for the complex (3.5)).

(ii) For the complex (3.4), the cohomology groups at levels $\Omega^{0}, \operatorname{Ker}\left(d_{J}^{-}\right), \Omega^{3}, \Omega^{4}$ are the usual de Rham cohomology groups $H^{i}(M, \mathbb{R})$ for $i=0,1,3,4$, respectively.

(iii) For the complex (3.5), the cohomology groups at levels $\Omega^{0}, \operatorname{Ker}\left(d_{J}^{+}\right), \Omega^{4}$ are the usual de Rham cohomology groups $H^{i}(M, \mathbb{R})$ for $i=0,1,4$, respectively. At the $\Omega^{3}$-level, the cohomology group of the complex (3.5) is given by $\mathcal{Z}^{3} / d \Omega_{J}^{-}$, so it is infinite dimensional, by Proposition 3.1.

Remark 3.7. All statements of Proposition 3.6 are still true in higher dimensions, except those about the cohomology at the $\Omega^{3}$-level which use Proposition 3.1. Note also that for a compact four-manifold $\operatorname{Ker}\left(d_{J}^{+}\right)=\mathcal{Z}^{1}$, so the second differential in the complex (3.5) is just the zero map.

\section{Acknowledgments}

We are grateful to Tian-Jun Li and the referees for useful suggestions about this note. The second author is partially supported by an AMS-Simons travel grant.

\section{References}

[1] D. Angella and A. Tomassini, On cohomological decomposition of almost-complex manifolds and deformations, J. Symplectic Geom. 9 (2011), 403-428.

[2] S.K. Donaldson, Two-forms on four-manifolds and elliptic equations, Inspired by S. S. Chern, 153-172, Nankai Tracts Math., 11, World Sci. Publ., Hackensack, NJ, 2006.

[3] T. Draghici, T.-J. Li and W. Zhang, Symplectic forms and cohomology decomposition of almost complex 4-manifolds, Int. Math. Res. Notices 2010 (2010), 1-17.

[4] T. Draghici, T.-J. Li and W. Zhang, On the J-anti-invariant cohomology of almost complex 4-manifolds, arXiv:1104.2511, Quart. J. Math., first published online December 6, 2011 doi:10.1093/qmath/har034.

[5] T. Draghici, T.-J. Li and W. Zhang, Geometry of tamed almost complex structures on 4dimensional manifolds, in Proceedings of ICCM 2010, AMS/IP Studies in Advanced Mathematics Vol. 51, 2012, 233-251.

[6] N. Enrietti, A. Fino, and L. Vezzoni, Tamed symplectic forms and SKT metrics, J. Symplectic Geom. 10 (2012), 203-224.

[7] A. Fino, Y.Y. Li, S. Salamon, and L. Vezzoni, The Calabi-Yau equation on 4-manifolds over 2-tori, arXiv:1103.3995. 
[8] A. Fino and A. Tomassini, On some cohomological properties of almost complex manifolds, J. Geom. Anal. 20 (2010), 107-131.

[9] M. Gromov, Pseudoholomorphic curves in symplectic manifolds, Invent. Math. 82(2) (1985), $307-347$.

[10] M. Lejmi, Strictly nearly Kähler 6-manifolds are not compatible with symplectic forms, C. R. Math. Acad. Sci. Paris 343(11-12) (2006), 759-762.

[11] T.-J. Li and A. Tomassini, Almost Kähler structures on four dimensional unimodular Lie algebras, J. Geom. Phys. 62 (2012), 1714-1731.

[12] T.-J. Li and W. Zhang, Comparing tamed and compatible symplectic cones and cohomological properties of almost complex manifolds, Commun. Anal. Geom. 17(4) (2009), 651-683.

[13] T.-J. Li and W. Zhang, Almost Kähler forms on rational 4-manifolds, preprint.

[14] T. Peternell, Algebraicity criteria for compact complex manifolds, Math. Ann. 275(4) (1986), 653-672.

[15] J. Streets and G. Tian, A Parabolic flow of pluriclosed metrics, Int. Math. Res. Not. 2010 (2010), 3101-3133.

[16] C. Taubes, Tamed to compatible: symplectic forms via moduli space integration, J. Symplectic Geom. 9 (2011), 161-250.

[17] C. Taubes, SW $\Longrightarrow$ Gr: from the Seiberg-Witten equations to pseudo-holomorphic curves, J. Amer. Math. Soc. 9(3) (1996), 845-918.

[18] V. Tosatti and B. Weinkove, The Calabi-Yau equation, symplectic forms and almost complex structures, in Geometry and Analysis, Vol. I, 475-493, Adv. Lect. Math. (ALM)17, International Press, 2011; arXiv:0901.1501.

[19] V. Tosatti and B. Weinkove, S.T. Yau, Taming symplectic forms and the Calabi-Yau equation, Proc. Lond. Math. Soc. (3) 97(2) (2008), 401-424.

[20] V. Tosatti and B. Weinkove, The Calabi-Yau equation on the Kodaira-Thurston manifold, J. Inst. Math. Jussieu 10(2) (2011), 437-447.

[21] B. Weinkove, The Calabi-Yau equations on almost Kähler manifolds, J. Differential Geometry, vol 76 (2)(2007), 317-349.

Department of Mathematics, Florida International University, Miami, FL 33199, USA

E-mail address: draghici@fiu.edu

Department of Mathematics, University of Michigan, Ann Arbor, Mi 48109, USA

E-mail address: wyzhang@umich.edu 
